\title{
CONTROL OF A TELEOPERATOR SYSTEM WITH REDUNDANCY BASED ON PASSIVITY CONDITIONS*
}

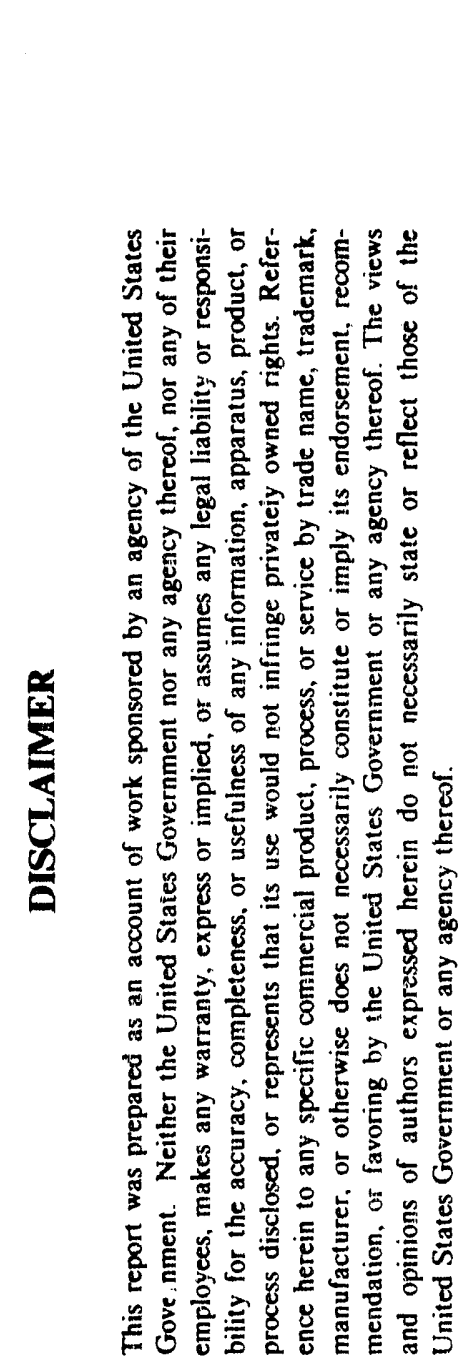

\author{
J. F. Jansen and R. L. Kress \\ Oak Ridge National Laboratory $†$ \\ P. O. Box 2008 \\ Oak Ridge, Tennessee 37831
}

CONF-910451--12

DE91 007679

\author{
To be presented at the \\ 1991 IEEE International Conference on \\ Robotics and Automation \\ Sacramento, California \\ April 7-12, 1991
}

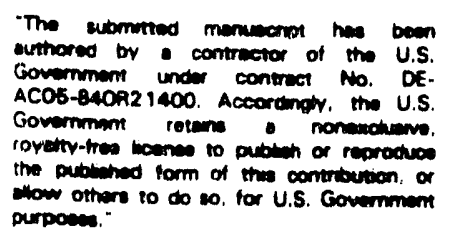

"Research sponsored by the U.S. Department of Energy.

+ Managed by Martin Marietta Energy Systems, Inc., for the U.S. Department of Energy under contract DE-AC05-84OR21400. 


\title{
CONTROL OF A TELEOPERATOR SYSTEM WITH REDUNDANCY BASED ON PASSIVITY CONDITIONS*
}

\author{
J. F. Jansen \\ R. L. Kress \\ Oak Ridge National Laboratory $†$ \\ Robotics and Process Systems Division
}

P. O. Box 2008

Oak Ridge, Tennessee 37831

\begin{abstract}
The purpose of this paper is to examine how a stiffness controller can be used to control a teleoperator system. having a redundant degree-of-freedom (dof) slave manipulator and a dissimilar master. No path planning was allowed because the uncertainty of the environment and computational limitations. The proposed controller was implemented on the 7-dof Center for Engineering Systems Advanced Research Manipulator (CESARm) slave manipulator with a 6-dof Kraft master manipulator. The overall performance of this scheme and future directions are discussed.
\end{abstract}

\section{Nomenclature}

\begin{tabular}{|c|c|}
\hline n & $=$ number of dofs of the manipulator \\
\hline$C(q, q)$ & $=$ Coriolis and centrifugal terms $(n \times n)$ \\
\hline$F_{\text {ext }}$ & $=$ contact force/torque vector $(6 \times 1)$ \\
\hline $\mathrm{G}_{\mathrm{m}}$ & $=$ master transfer function matrix \\
\hline $\mathrm{G}_{\mathrm{s}}$ & $=$ slave transfer function matrix \\
\hline $\mathrm{G}_{\mathrm{s}-\mathrm{red}}$ & $=$ positive semi-definite matrix \\
\hline$G_{\text {si }}$ & $\begin{array}{l}=\text { transfer functions for each of the cartesian } \\
\text { directions }\end{array}$ \\
\hline $\mathrm{H}$ & $\begin{array}{l}=\text { Jacobian of constraints or performance } \\
\text { criteria }\end{array}$ \\
\hline I & = identity matrix \\
\hline$J(q)$ & $=$ manipulator Jacobian $(6 \times n)$ \\
\hline$J^{\#}$ & $\begin{aligned}= & \text { pseudoinverse of the manipulator } \\
& \text { Jacobian, } J\end{aligned}$ \\
\hline $\mathrm{J}_{\mathrm{m},} \mathrm{J}_{\mathrm{s}}$ & $=$ master and slave Jacobian, respectively \\
\hline$J_{\text {red }}$ & $\begin{aligned}= & \text { redundancy Jacobian relating elbow } \\
& \text { cartesian motions to joint motion }\end{aligned}$ \\
\hline $\begin{array}{l}K_{s-\text { red }} \\
M(q)\end{array}$ & $\begin{array}{l}=\text { constant positive-definite matrix } \\
=\text { inertia matrix }(n \times n)\end{array}$ \\
\hline
\end{tabular}

\footnotetext{
* Research sponsored by the U.S. Department of Energy

Thanaged by Martin Marietta Energy Systems, Inc., for the U.S. Department of Energy under contract DE AC05-840R21400.
}

$$
\begin{array}{ll}
\mathrm{q}, \dot{\mathrm{q}} & =\begin{array}{l}
\text { generalized joint coordinates and } \\
\text { velocities, }(\mathrm{n} \times 1)
\end{array} \\
\mathrm{T} & =\text { time } \\
\widehat{\mathrm{x}}_{\mathrm{elb}} & =\text { eibow Cartesian position } \\
\widehat{\mathrm{x}}_{\mathrm{elb}}^{\text {des }} & =\text { desired elbow Cartesian position } \\
\mathrm{x}_{\mathrm{m}}, \mathrm{x}_{\mathbf{s}} & =\text { master and slave position, respectively } \\
\gamma & =\text { arbitrary real number } \\
\theta_{0} & =\text { joint space point } \\
\tau & =\text { joint force/torque vector }(\mathrm{n} \times 1) \\
\tau_{\text {fric }} & =\text { frictional force/torque vector }(\mathrm{n} \times 1) \\
\tau_{\mathrm{grav}} & =\text { gravitational force/torque vector }(\mathrm{n} \times 1) \\
\tau_{\text {red }} & =\text { redundancy torque }(\mathrm{n} \times 1)
\end{array}
$$

\section{Introduction}

\subsection{Brief History and Overview of Teleoperation}

In the late 1940s, R. C. Goertz and his colleagues at Argonne National Laboratory developed one of the earliest recognizable mechanical master/slave manipulators-first without force reflection and later with force-reflecting capabilities. In the early 1950 s, Goertz and his colleagues developed an electric master/slave manipulator in which each slave-joint servo was tied directly to the master-joint servo because both the mast ar and slave were kinematically similar [Goertz, 54]. The control structure for these manipulators was the classical position-position controller. A positional difference between the slave and the master is reflected back as a drive signal to the master to push the human operator away from the object. The controller implementation was purely analog based on ac servo technology. The position-position control scheme has been the basic controller for almost all master/slave manipulators used by industry up to the present time. with the only modification being the convers on of the 
analog controller to a digital controller. The primary objective of a teleoperated system is to reflect the impedance of the operator to the environment. This objective is accomplished by means of large-jointstiffness coupling between the master and slave. Positional accuracy has historically been of secondary importance for two reasons. The first is that human operators are poor positional controllers. Steady-state master and slave positions can easily differ by a few inches without the human operator's noticing the difference. Only changes in positional difference are noted by the human operawr. The second reason is that to reflect the operator's impedance to the environment, the joint friction was minimized by accepting high levels of backlash in the gearing/transmission systems to reduce contact friction, thus reducing transmission losses but increasing positional error. It should be noted that a position-position control scheme is a passive type of controller.

\subsection{Goals and Design Objectives}

The primary goal for this project was to determine if a redundant teleoperator system with dissimilar kinematics and a redundant dof for the slave manipulator could be controlled in an unknown and a potentially dangerous environment. No path preview was allowed because the human operator would be making decisions rapidly, and only modest computation power was available. To resolve the redundancy issue, an elbow sensor would be placed on the elbow of the human operator. This sensor would give a signal proportional to the positional change of the user and would be used to control the elbow of the 7-dof CESARm slave. Because of funding limitations, an elbow sensor was not available; however, the elbow position change of the user was implemented as an input command. This implementation will be clarified later in this paper.

\subsection{Basic Problems with Dissimilar Kinematic Teleoperators}

When the master and slave are not kinematically similar and the slave has a redundant dof, the design of the controller is particularly difficult. Three major issues are associated with this control problem: (1) orientation representation, (2) accurate force refection, and (3) redundancy resolution. The first two issues have been addressed by the authors in a previous paper [Jansen, 90]; therefore, only the last issue will be addressed in this paper.

Redundancy resolution has been an active area of research for a number of years. Because of real-time implementation issues associated with a teleoperated system, only redundancy resolution schemes using local optimization methods are relevant. Two schemes dominate: resolved motion rate control (RMRC) and resolved acceleration control techniques [Nenchev, 89]. Both of these techniques require utilization of a pseudoinverse when the slave manipulatcr has redundant dofs. Problems with pseuduirverse techniques are: (1) nonrepeatability [Klein, 83] of starting positions of a manipulator after transversing a fix closed path, (2) potential instability due to the fact that many teleoperator tasks are close to the workspace boundary (and therefore close to singularity points) and the slave must make contact with objects in an unstructured environment, and (3) difficulty in selection of local optimization criteria that physically relate to the specific tasks being performed. To circurnvent these problems and to exploit the fundamental difference between a robot and a teleoperator, a redundancy resolution scheme based on passivity and stiffness concepts will be discussed next.

\section{Redundancy Resolution}

\subsection{Basic Idea}

The slave manipulator which is being considered in this paper has 7 dof. Six dof are required to obtain an arbitrary position and orientation of a manipulator end effector. A 7-dof slave manipulator is desirable because the additional (or redundant) dof allows for numerous manipulator configurations for a given end-effector position and orientation, thus permitting the manipulator to reach around obstacles to perform a task, to avoid joint limits, or to optimize its configuration in some other manner. The slave manipulator will incorporate a stiffness controller [Salisbury, 80 ] and [Miyazaki, 86]. The basic idea is to add additional constraints to the system so that the end-effector Jacobian is extended to have full rank. Previous researchers [Oh, 84; Colbaugh, 89] have applied the criterion that additional constraints cannot create self-motion, where self-motion is defined as motion not affecting the position or orientation of the end-effector [Baillieul, 86].

The constraints are to be set such that: (1) a small amount of positional error will occur, (2) passivity concitions will be applied to assure stability, and (3) utilization of the redundant dof is accomplished by means of an elbow sensor on the human operator.

\subsection{Passivity}

Passivity simply means that the energy input to a component or system must be greater than some negative number for all time, that is,

$$
\int_{0}^{\mathrm{T}} \mathrm{q}^{\mathrm{T}} \tau \mathrm{dt} \geq-\gamma^{2} \forall \mathrm{T} \geq 0 .
$$


If the power flow is reversed, then the inequality is reversed, and the nerative sign on the right is removed. Passivity can be shown for a mechanical manipulator. A sketch of the proof can be found in [Asada, 86], and only the results will be shown. The dynamic equations of motion for a rigid-bodied link manipulators [Khatib, 87] is

$$
\begin{aligned}
& \tau=M(q) \ddot{q}+C(q, \dot{q}) \dot{q}+ \\
& J(q)^{T} F_{\text {ext }}+\tau_{\text {grav }}+\tau_{\text {fric }}
\end{aligned}
$$

For the rest of this paper, the functional dependency of $\mathrm{M}, \mathrm{C}$, and $\mathrm{J}$ will be dropped to reduce notational clutter. Subscripts $m$ and $s$ designate either master parameters or slave parameters, respectively. Passivity gives the following relationship:

$$
\begin{gathered}
\int_{0}^{T} \dot{q}^{T} \tau d t \geq-\dot{q}(0)^{T} M(q) \dot{q}(0)+ \\
\int_{0}^{T}\left[\dot{q}^{T} J(q)^{T} F_{\text {ext }}+\dot{q}^{T} \tau_{\text {grav }}+\dot{q}^{T} \tau_{\text {fric }}\right] d t
\end{gathered}
$$

The human operator and the environment will be assumed to be modeled as passive systems. There are limitations with representing the operator and environment with passive models [Anderson, 89]; nevertheless, investigations using a passive model build confidence with the proposed control techniques. Because the external environment is assumed passive, the first term in the integral of $\mathrm{Eq}$. (3) satisfies the passivity condition-likewise, for the last term inside the

integral. Exploiting the fact that $\tau_{\mathrm{gmv}}=-\nabla \mathrm{V}$, where $\mathrm{V}$ is a potential function, passivity for Eq. (3) can be shown.

R. Anderson [Anderson, 90] defined a control law which satisfies the passivity constraint as a passive control law. Both the master and slave controllers are designed to be passive controllers. Because it has been shown by numerous researchers [Asada, 86] that a stiffness controller [Salisbury, 80; Miyazaki, 86] is a passive controller, both the master and slave controller will be modified stiffness controllers. Passivity has been proposed for a nonredundant teleoperated system by G. J. Raju [Raju, 89] a.-d others. Impedance control [Hogan, 85] was not selected because of computational limitations of the hardware used by the authors. A further hardware limitation was the lack of velocity signals available at the computer.

\section{Controller}

\subsection{Stiffness Controller}

The master manipulator incorporates a modified stiffness controller. The master torque signal is

$$
\tau_{\mathrm{m}}=\mathrm{J}_{\mathrm{m}}^{\mathrm{T}}\left[\mathrm{G}_{\mathrm{m}}\left(\mathrm{x}_{\mathrm{s}}-\mathrm{x}_{\mathrm{m}}\right)\right]
$$

The slave manipulator incorporates a modified stiffness controller. The slave torque signal is

$$
\tau_{\mathrm{s}}=\mathrm{J}_{\mathrm{s}}^{\mathrm{T}}\left[\mathrm{G}_{\mathrm{s}}\left(\mathrm{x}_{\mathrm{m}}-\mathrm{x}_{\mathrm{s}}\right)\right]+\tau_{\mathrm{red}} \text {. }
$$

\subsection{Stability Based on passivity}

Figure 1 shows the bond graph of a teleoperator system. Power flows and causality markers are shown by the half arrows and causality strokes, respectively.

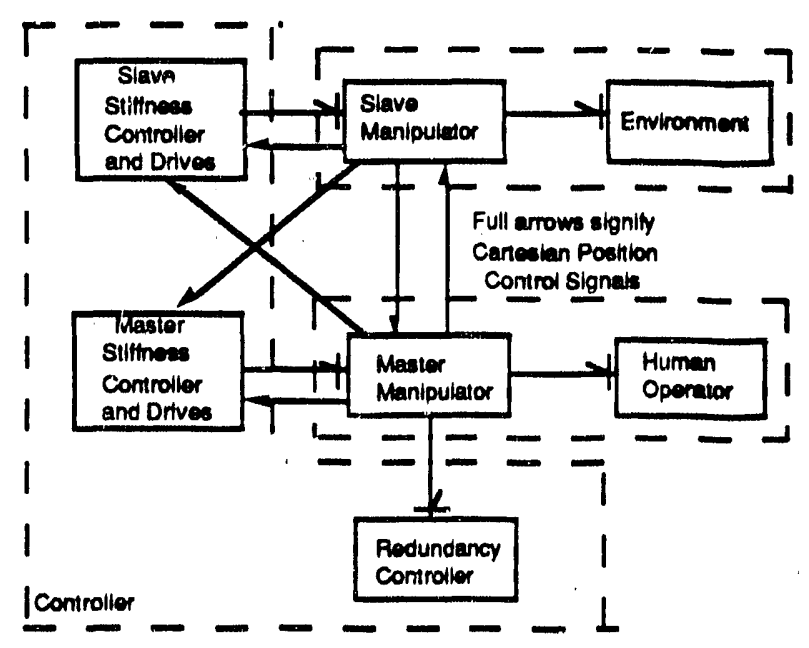

Fig. 1. Bond graph of a teleoperator system.

Based on the idea of hyperstability, if the controllers can be shown to be passive, then the whole system will be stable because a linear time-invarient system which is passive also satisfies the positive realness conditions. From B. D. O. Anderson [Anderson, 68], a rational transfer-function $G(s)$ is positive real if the following conditions are met: (1) all elements of $G(s)$ are analytic in $\operatorname{Re}[s]=0$; (2) the denominator polynomial of $G$ is a Hurwitz polynomial or may have zeros on the $j$ axis; that is, $G$ must be analytic in the right half-plane; (3) if $G$ has poles on the $\mathrm{j}$ axis, these poles must be simple and have real and positive residues; and (4) the condition $\operatorname{Re}[G(j \omega)] \geq 0$ for all $\omega$ must be satisfied.

The controllers will be designed to have the property of positive realness. If each controller satisfies the positive realness condition, then based on Popov's hyperstability condition, the overall system is stable. The problem becomes one of ensuring that

$$
\int_{0}^{T}\left(\dot{q}_{m}^{T} \tau_{m}+\dot{q}_{s}^{T} \tau_{s}\right) d t \leq \gamma_{1}^{2} \forall T>0 .
$$

The dashed boxes in Fig. 1 lumps the master and slave controllers together to make them separate passive systems. Power is the input in the definition of 
Eq. (3), whereas power is the output of the master and slave controllers of Fig. 1; therefore, the inequality and sign have been reversed. The slave and master dashed boxes have been shown to be passive; thus,

$$
\begin{gathered}
-\gamma_{2}^{2} \leq \int_{0}^{T}\left(q_{m}^{T} \tau_{\mathrm{m}}\right) d t \quad \forall \mathrm{T}>0 \text { and } \\
-\gamma_{3}^{2} \leq \int_{0}^{T}\left(q_{s}^{T} \tau_{s}\right) d t \quad \forall T>0,
\end{gathered}
$$

where $\gamma_{2}$ and $\gamma_{3}$ are arbitrary real constants. Using Eqs. (7) and (8), Eq. (6) can be rewritten as

$\int_{0}^{T}\left(\kappa q_{m}^{T} \tau_{m}+q_{s}^{T} \tau_{s}\right) d t \leq \gamma_{4}^{2} \forall T>0$,

where $K$ is any arbitrary real positive constant and $\gamma_{4}$ is an arbitrary real constant. Inserting the master and slave controller equations into Eq. (9) results in

$\int_{0}^{T}\left\{\begin{array}{c}\kappa \dot{q}_{m}^{T}\left[J_{m}^{T} G_{m}\left(x_{s}-x_{m}\right)\right]+ \\ \dot{q}_{s}^{T}\left[J_{s}^{T} G_{s}\left(x_{m}-x_{s}\right)+\tau_{r o d}\right]\end{array}\right\} d t \leq \gamma_{4}^{2} \forall T>0$

Using the relationship that $J_{m} \dot{q}_{m}=\dot{x}_{m}, J_{s} q_{s}=\dot{x}_{s}$, $G_{s}=\kappa G_{m}$, and $z=\dot{x}_{s}-\dot{x}_{m}$, the following two conditions are required:

$$
\begin{aligned}
& \int_{0}^{T} z^{T} G_{s}\left|\int_{0}^{t} z d \xi\right| d t \geq-\gamma_{s}^{2} \forall T>0 \\
& \text { and } \quad \int_{0}^{T} q_{s}^{T} \tau_{\text {red }} d t \leq \gamma_{6}^{2} \forall T>0
\end{aligned}
$$

where $\gamma_{5}$ and $\gamma_{6}$ are arbitrary real constants because $\gamma_{4}$ is an arbitrary real constant. The design goal is to determine $G_{s}$ and $\tau_{\text {red }}$ such that Eqs. (11a) and (11b) are satisfied. Obviously, if the conditions stated in Eqs. (11a) and (11b) are satisfied, then the condition stated in Eq. (10) is satisfied. Based on the positive real lemma, the condition stated in Eq. (11a) will be satisfied if

$$
\frac{G_{\mathrm{s}}(\mathrm{s})}{\mathrm{s}}=\mathbf{L}\left\{\mathrm{G}_{\mathrm{s}}\left(\int_{0}^{\mathrm{t}}\right)\right\}
$$

is a positive real matrix, where $\mathcal{L}$ is the Laplace operator. The next two sections will discuss how to satisfy the rediundancy condition of Eq. (11b) and the condition specified by Eq. (12).

\subsection{Redundancy Condition Satisfaction}

The basic idea is shown in Fig. 2. A spring is added to the elbow. As mentioned before, an elbow sensor on the human operator is anticipated to provide the datum position to the slave position. The human operator will have direct control over each of the dof of the slave manipulator. A fictitious, low-stiffiness spring is attached between the elbow and the ground point. This elbow spring is usually antagonistic to the hand spring as shown in the figure. The hand- spring stiffness is set to a much larger value than the elbow spring stiffness to reduce the positional error. This is discussed in the next section.

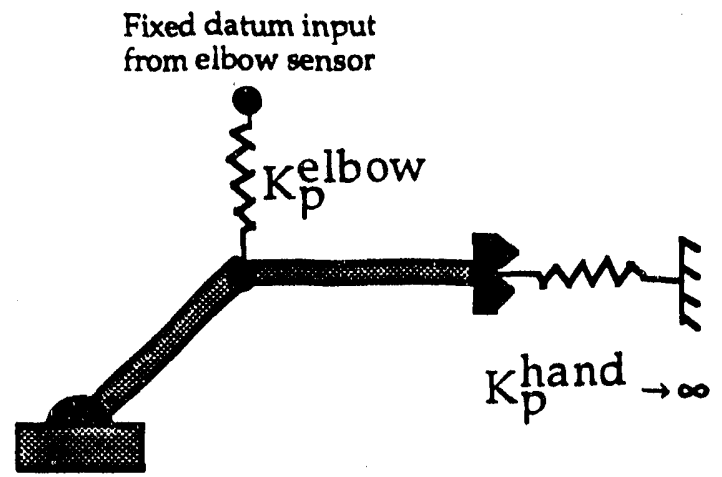

Fig. 2. Elbow constraint.

Adding a constraint to the elbow can be conveniently expressed as

$$
\tau_{\text {red }}=J_{\text {red }}^{T}\left[G_{s-\text { red }}\left(\widehat{x}_{\text {elb }}^{\text {des }}-\widehat{x}_{\text {elb }}\right)\right] \text {. }
$$

$J_{\text {red }}$ has the property that $J_{\text {red }} q_{s}=\tilde{I} \dot{\hat{x}}_{\text {elb }}$, where $\tilde{I}=\left[\begin{array}{ll}I_{3} & O_{3}\end{array}\right]^{\mathrm{T}}, I_{3}=3 \times 3$ identity matrix and $O_{3}=3 \times 3$ zero matrix. Substituting Eq. (13) into Eq. (11b) results in

$$
\int_{0}^{\mathrm{T}} \dot{\mathrm{v}}_{\mathrm{elb}}^{\mathrm{T}} \mathrm{G}_{\mathrm{s}-\mathrm{elb}}(\mathrm{v}) \mathrm{dt} \geq-\gamma_{6}^{2} \forall \mathrm{T}>0,
$$

where $v=\widehat{x}_{e l b}-\widehat{x}_{e l b}^{\text {des }}$ and $\widehat{x}_{e l b}^{\text {des }}$ is fixed. Similar to the previous case, the condition stated by Eq. (14) will be satisfied if

$$
\frac{G_{s-\text { red }}(s)}{s}=\mathcal{L}\left\{G_{s-\text { red }}\left(\int_{0}^{t}\right)\right\}
$$

is a positive real matrix. Because of high levels of friction and the low values of elbow stiffness that were

required, $G_{s-r e d}$ was set to $K_{s-\text { red, a constant positive- }}$ definite matrix.

\section{$3.4 G_{s}$ Selection}

The master and slave transfer function matrices, for simplicity, are both selected to be diagonal matrices. Only the slave compensator equation will be written because the master and slave compensators are proportional. Thus, 


$$
\begin{aligned}
& \mathfrak{a}_{\mathrm{s}}(\mathrm{s})=\operatorname{diag}\left[\mathfrak{G}_{\mathrm{s} 1}(\mathrm{~s}) \mathfrak{G}_{\mathrm{s} 2}(\mathrm{~s}) \mathfrak{G}_{\mathrm{s} 3}(\mathrm{~s})\right. \\
& \left.\mathfrak{G}_{\mathrm{s} 4}(\mathrm{~s}) \mathfrak{G}_{\mathrm{s} 5}(\mathrm{~s}) \mathfrak{G}_{\mathrm{s} 6}(\mathrm{~s})\right] \text {. }
\end{aligned}
$$

The objectives of the joint servo compensators are to stiffen the joint servos (i.e., increase static accuracy), improve system stability margins, and improve system insensitivity to high-frequency noise. Only the static accuracy will be addressed in this paper because a detailed discussion of the other two objectives can be found in [Chen, 89].

A lag-lead compensator can meet the previously mentioned objectives. A lag-lead compensator is given by

$$
\mathcal{G}_{\mathrm{si}}(s)=K_{\mathrm{s}} \frac{\left(s+\alpha_{1}\right)\left(s+\alpha_{2}\right)}{\left(s+\beta_{1}\right)\left(s+\beta_{2}\right)},
$$

where $\alpha_{1}, \alpha_{2}, \beta_{1}$, and $\beta_{2}$ are corner frequencies with $0<\beta_{1}<\alpha_{1}<\alpha_{2}<\beta_{2}$ and $K_{s}$ is a positive gain term. The lag-lead compensator can be designed to minimize the dynamic range of the joint servo. Because the frequency content of human operator motion is less than $1 \mathrm{~Hz}$ for most real tasks [Noakes, 90], the frequency response of a teleoperator system will not be significantly affected by this type of compensator.

To satisfy the positive real condition, all four previously discussed conditions must be satisfied. The first three can be easily shown to be satisfied. Condition 4 can be shown to be satisfied if $\operatorname{Re}\left[\mathfrak{G}_{s i}(j \omega) / j \omega\right] \geq 0$, which is equivalent to

$$
\begin{gathered}
\left(\beta_{1}-\alpha_{1}\right)\left(\omega^{2}+\alpha_{2} \beta_{2}\right)+\left(\beta_{2}-\alpha_{2}\right) \\
\left(\omega^{2}+\alpha_{1} \beta_{1}\right) \geq 0, \forall \omega .
\end{gathered}
$$

Using the approximation that $s \cong \frac{2}{T}\left(\frac{z-1}{z+1}\right), G_{s_{i}}(z)$ becomes

$$
G_{s i}(z)=K_{s}^{\prime} \frac{\left(z+\alpha_{1}^{\prime}\right)\left(z+\alpha_{2}^{\prime}\right)}{\left(z+\beta_{1}^{\prime}\right)\left(z+\beta_{2}^{\prime}\right)} \text {. }
$$

where

$$
\begin{gathered}
\alpha_{1}^{\prime}=\frac{\alpha_{1} T-2}{2+\alpha_{1} T}, \quad \alpha_{2}^{\prime}=\frac{\alpha_{2} T-2}{2+\alpha_{2} T^{\prime}}, \quad \beta_{2}^{\prime}=\frac{\beta_{2} T-2}{2+\beta_{2} T} \\
K_{s}^{\prime}=K_{s} \frac{\left(2+\alpha_{1} T\right)\left(2+\alpha_{2} T\right)}{\left(2+\beta_{1} T\right)\left(2+\beta_{2} T\right)}
\end{gathered}
$$

The stiffness of the lag-lead compensator must be much larger than the stiffness of the elbow constraint. At $\omega=0$, the stiffness of the lag-lead compensator is

$$
\text { stiffness }=K_{3} \frac{\alpha_{1} \alpha_{2}}{\beta_{1} \beta_{2}} .
$$

This value must be selected to be a fairly large number to satisfy the stiffness requirements. It should be noted

that high levels of stiffness are required for $\boldsymbol{C}_{\mathbf{3}}$ a s compared to the elbow to minimize the steady-state positional error. Because of the passivity constraint of Eq. (18) and to limit cycles [Gelb, 68] resulting from unmodeled dynamics, infinite stiffness is obviously not possible; however, we were able to selut values that increased the overall steady-state accuracy, or stiffness, 50 times higher than with a standard proportionalderivative controller.

\section{Application to CESARm/Kraft Manipulators}

\subsection{Implementation}

The master controller was implemented on the Kraft master arm. The Kraft KMC 9100-MC is a lightweight 6-dof master arm designed, manufactured. and sold by Kraft Telerobotics, Inc., of Overland Park, Kansas. Position is measured at each joint by potentiometers. The first five joints are actuated by ac servomotors for force feedback. Wrist roll is not actuated. The Kraft arm is sold with the KMC 9100 S electronics interface unit.

The slave algorithm is implemented on the CESARm [Babcock, 87], which is a customdesigned 7-dof arm and is not a production-grade manipulator but a research manipulator used to determine feasibility and performance of various redundancy algorithms. Its joints are (from base to gripper) waist, shoulder pitch, shoulder roll, elbow, wrist pitch, wrist yaw, and wrist roll. The waist, shoulder pitch, and shoulder roll are actuated with dc servomotors coupled to gear reducers. The elbow is actuated by a dc servomotor coupled through a 4-bar linkage. Wrist pitch and yaw are actuated by dc servomotors connected through cables to a 2-dof differential. Wrist roll is actuated by a dc servomotor connected via cables. Position is measured at each motor by optical encoders, and velocity is measured for analog feedback by tachometers mounted on the motors. Three components of force and three components of torque are measured at the CESARm wrist by a $\mathrm{JR}^{3}$ universal force sensor communicating through a parallel interface (not used for this study). Both the CESARm and the Kraft are shown in Fig. 3.

The master and slave control algorithms were programmed in $\mathrm{C}$ language and implemented on three Motorola 68020 s having 68881 floating point coprocessors. The algorithms were partitioned as 


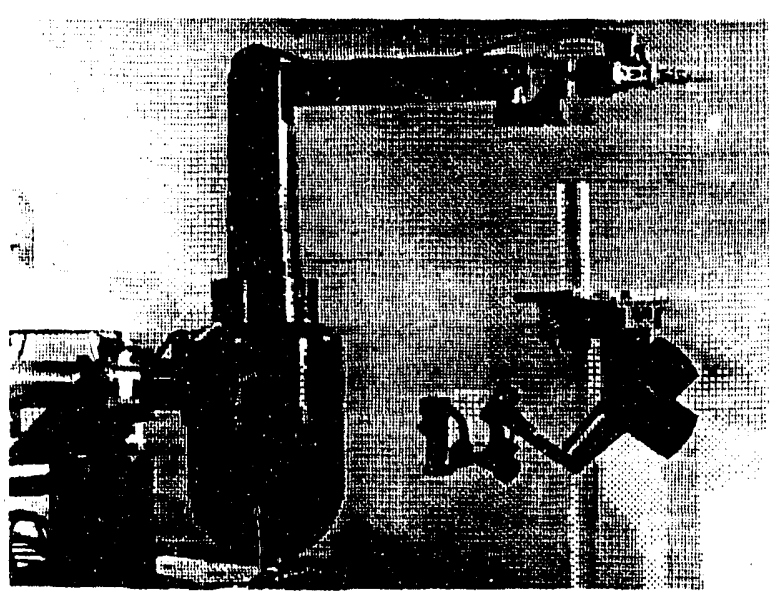

Fig. 3 CESARm and Kraft manipulators.

follows. CPU 1 had all low-level routines. This included low-level control routines (e.g., sending motor signals, enabling motors and brakes, reading encoders, etc.), simple joint and cable limit checks, and input/output routines. CPU 2 had the stiffness and redundancy resolution algorithms and an obstacle avoidance routine. CPU 3 had the master stiffness routine, the $\mathrm{JR}^{3}$ force/torque sensor routine, and master and $\mathrm{JR}^{3}$ communications.

The master controller is currently implemented on the 6-dof, force-reflecting Kraft master manipulator and runs at a loop rate of $-20 \mathrm{~Hz}$. The relatively low loop rate of the master side of the controller is a result of high communications overhead between the CPU and the Kraft control electronics. The slave algorithm is implemented on the 7-dof CESARm and runs at a loop rate of $\sim 100 \mathrm{~Hz}$.

\subsection{Experimental results}

The controller was tested on the CESARm performing a number of simple tasks. These included slow and fast motion in free space, motion at the limit of the operational space, contact with rigid objects (such as steel) and non-rigid objects (wood and rubber materials), and simple stacking operations. The arm remained stable during all modes of operations with fair (because of time limitations only a crude visual measurement was possible) transient response. Each of the joint servos had roughly $1.5-$ to $2-\mathrm{Hz}$. bandwidth. The low bandwidth is due primarily to the compliance in the cable drives and the gear boxes. Positional accuracy bounds were measured by sending a signal to the slave requesting the slave to move back and forth along a straight line of length $0.4 \mathrm{~m}$ with a 5-s. period. The peak positional errors were below $1.25 \mathrm{~cm}$ for an elbow spring deflection of roughly $0.2 \mathrm{~m}$.

\section{Conclusion and Summary}

Controlling a redundant manipulator has been and still is a very challenging problem. This paper addressed the problem of controlling a redundant teleoperator system with dissimilar kinematics and a redundant dof for the slave manipulator in an unknown environment. No path preview was allowed, and only modest computation power was available. A stiffiness type of controller was selected because it could be easily modified to be passive and avoided the use of pseudoinverse methods and, more importantly, the stiffness of the slave could be easily changed. Exploiting the fact that positional accuracy is not the primary goal of a teleoperator system, small positional errors were allowed to provide a fairly simple and physically appealing control. The modified stiffness controller worked well on the Kraft master and the CESARm slave manipulator in a number of different teleoperated tasks.

Future work is envisioned using redundancy criteria different from the elbow constraint condition along with consideration of human factors issues in the selection of redundancy conditions. An elbow- tracking sensor could be implemented and tested. Replacing the stiffness controller with impedance control schemes [Hogan, 85] is also being examined.

\section{Acknowledgements}

The authors wish to thank Mike Kedl and Phil L. Butler for their diligent effort in providing software and hardware support during this research.

\section{References}

[Anderson, 68] Anderson, B. D. O., "A Simplified Viewpoint of Hyperstability," IEEE Trans. on Automatic Control, pp. 292-294, 1968.

[Anderson, 89] Anderson, R., and Spong, M., "Asymptotic Stability for Force Reflecting Teleoperators with Time Delay," 1989 IEEE International Conference on Robotics and Automation, Vol. 3, May 1990, pp. 1618-1625.

[Anderson, 90] Anderson, R., "Dynamic Damping Control: Implementation Issues and Simulation Results," 1990 IEEE International Conference on Robotics and Automation, Vol. 1, May 1990, pp. 6877.

[Asada, 86] Asada, H., and Slotine, J.-J. E., Robot Analysis and Control John Wiley and Sons, New York, NY, 1986. 
[Babcock, 87] Babcock, S. M., Hamel, W. R., and Killough, S. M., "Advanced Manipulation for Autonomous Mobile Robots," Proceedings of ANS International Topical Meeting on Remote Systems and Robotics in Hostile Environments, Pasco, Washington, March 29-April 2, 1987, pp. 290-297.

[Baillieul, 86] Baillieul, J., "Avoiding Obstacles and Resolving Kinematic Redundancy," 1986 IEEE International Conference on Robotics and Automation, Vol. 3, April 1986, pp. 1698-1704.

(Chen, 89] Chen, Y., 1989 IEEE Trans. on Robotics and Automation, Vol 5, No. 2, April 1989, pp. 174182.

[Colbaugh, 89] Colbaugh, R., Seraji, H., and Glass, K. L., "Obstacle Avoidance for Redundant Robots Using Configuration Control." J. of Robotic Systems Vol. 6, No. 6, pp. 721-744, 1989.

[Craig, 89] Craig, J. J., Introduction to Robotics: Mechanics and Control. Addison-Wesley, Reading, MA 1989.

[Gelb, 68] Gelb, A., and Velde, W. E. V, MultipleInput Describing Functions and Nonlinear System Desien, McGraw-Hill, Inc., New York, NY, 1968.

[Goertz, 54] Goertz, R. C., and Thompson, W. M., "Electronically Controlled Manipulator," Nucleonics, November 1954, pp. 46-47.

[Hogan, 85] Hogan, N., "Impedance Control: An Approach to Manipulation: Part 1 - 3 Theory," J. of Dynamic Systems, Measurement, and Control, Vol. 107, March 1985, pp. 1-24.

[Jansen, 90] Jansen, J. F., Kress, R. L., and Babcock, S. M., "Controller Design for a ForceReflecting Teleoperator with Kinematically Dissimilar Master and Slave," To be published in the J. of Dynamic Systems, Measurement, and Controi, , 1991.
[Khatib, 87] Khatib, O., "A Unified Approach for Motion and Force Control of Robot Manipulators: The Operational Space Formulation," IEEE J. of Robotics and Automation, Vol. RA-3, No. 1, February 1987, pp. 43-53.

[Klein, 83] Klein, C. A., and Huang, C. S., "Review of Pseudoinverse Control for Use with Kinematically Redundant Manipulators," IEEE Trans. Sys., Man, and Cyber., Vol. 13, 1983, pp. 245-250.

[Miyazaki, 86] Miyazaki, F., Matsubayashi, S., Yoshimi, T., and Arimoto, S., "A New Control Methodology Toward Advanced Teleoperation of Master-Slave Robot Systems," 1986 IEEE International Conference on Robotics and Automation, pp. 9971002.

[Nenchev, 89] Nenchev, Dragomir, "Redundancy Resolution Through Local Optimization: A Review," $J$. of Robotic Systems. Vol. 6, No. 6, pp. 769-798, 1989.

[Noakes, 90] Noakes, M. W., Oak Ridge National Laboratory, Personal communication, 1990.

[Oh, 84] Oh, S. Y., Orin, D., and Bach, M., "An inverse Kinematic solution for kinematically redundant Robot Manipulators," $J$. of Robotic Systems, 1, pp. 235-249, 1984.

[Salisbury, 80] Salisbury, J. K., "Active Stiffness Control of a Manipulator in Cartesian Coordinates", IEEE Conf. Decision and Control, Albuquerque, NM, November 1980, pp. 95-100.

[Raju, 89] Raju, G. J., Verghese, G. V., Sheridan, T. B., "Design Issues in 2-port Network Models of Bilateral Remote Manipulation," 1989 IEEE International Conference on Robotics and Automation, Vol. 3, May 1989, pp. 1316-1321. 

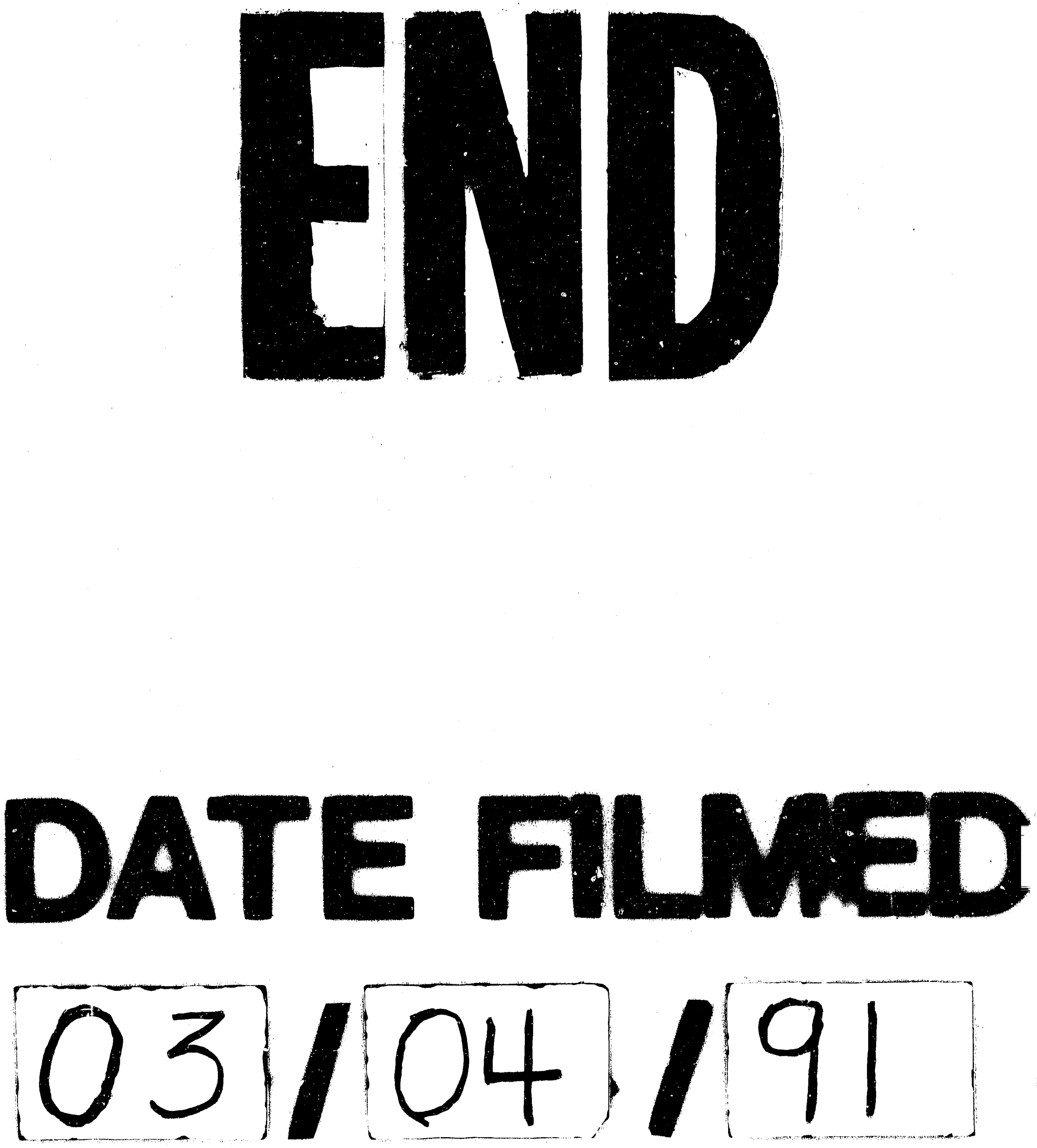
